\title{
Performance of low-resistivity single and dual-gap RPCs for LHCb
}

\author{
M. Adinolfi, G. Carboni, R. Messi, L. Pacciani, L. Paoluzi, E. Santovetti \\ Università degli Studi di Roma “Tor Vergata” and INFN, Roma, Italy
}

Presented by. G. Carboni

October 29, 1999

\begin{abstract}
RPCs are strong candidates for the outer regions of the LHCb muon detector. We have tested single-gap and dual-gap detectors built with low-resistivity phenolic plates $\left(\rho=9 \cdot 10^{9} \Omega \mathrm{cm}\right)$ and operated in avalanche mode. Measurements have been performed over a wide range of beam intensities and on the GIF at CERN. The results are presented and discussed, with special emphasis on the detection efficiency.
\end{abstract}

\section{Introduction}

The muon detector of experiment $\mathrm{LHCb}$ is made of 5 tracking stations, M1-M5. RPCs constitute a very attractive solution for equipping the outer region of stations M2-M5, located after the absorber, where the particle flux is relatively small [1]. RPCs have several advantages, namely very fast response, robustness and simplicity of construction, and lend themselves well to economical industrial production. Their weak point is the capability to work at high rate, but recently progresses have been done in this direction. In $\mathrm{LHCb}$, taking into account various safety factors, the detectors are required to withstand a maximum flux of $3 \mathrm{kHz} / \mathrm{cm}^{2}$ at $2 \mathrm{~m}$ from the beam axis. We have therefore studied the performances of single and doublegap RPCs under various rate conditions either on intense beams or on the Gamma Irradiation Facility (GIF) at CERN.

\section{The Detectors}

High-rate RPCs must be operated in avalanche mode to reduce the time for recharging the detector [2]. Moreover, since the characteristic time constant for recharge is directly proportional to the resistivity of the bakelite, this should be as small as practicable. In the detectors tested 
here we have used bakelite with resistivity of $9 \cdot 10^{9} \Omega \mathrm{cm}$ and thickness of $2 \mathrm{~mm}$, yielding $\tau \cong 5.6 \mathrm{~ms}$.

We built 3 types of detectors, all of $50 \times 50 \mathrm{~cm}^{2}$ dimensions and $2 \mathrm{~mm}$ gas gap: (a) singlegap (SRPC); (b) double-gap (DRPC) and (c) a triplet of single-gap. Round spacers (12 mm dia.) were glued between the bakelite plates at $10 \mathrm{~cm}$ distance along $x$ and $y$.

Types (a) and (c) were treated with linseed oil and had $3 \mathrm{~cm}$ wide strips on the two sides (X and $\mathrm{Y}$ readout). In (c) the corresponding strips in the triplet were precisely aligned. Type (b) had $2 \mathrm{~cm}$ strips. Following CMS design, the strip plane was sandwiched between the two gas gaps [3].

The electronics consisted of fast voltage amplifiers with a gain of 300, directly coupled to the strips. The strips were terminated on the other side by appropriate resistors. The amplifiers were followed by fast discriminators and multi-hit TDCs. A $1 \mathrm{~cm}$-pitch X-Y hodoscope was used to select the incoming beam. A gas system using mass flowmeters monitored precisely the gas mixture, normally consisting of $95 \% \mathrm{C}_{2} \mathrm{H}_{2} \mathrm{~F}_{4}, 4 \%$ isobutane and $1 \% \mathrm{SF}_{6}$ [4].

The dark current drawn by the chambers showed considerable differences between the single-gap and the double-gap. The SRPC current was well below $1 \mu \mathrm{A}$ whereas it was up to several tens of $\mu \mathrm{A}$ for the two gaps of the DRPC. The dark current in the DRPC gave a large random counting rate. We believe that the difference SRPC-DRPC was mainly due to the treatment with linseed oil in the SRPC. In fact, a subsequent treatment with oil on the DRPC resulted in a sizeable reduction of the current. However the dark current and the associated random rate did not affect adversely the performances of the detectors.

\section{Beam tests}

High-intensity beam data have been collected at the T11 pion beam at the CERN PS. The beam was approximately round in shape, with a FWHM of about $3 \mathrm{~cm}$. We took data at several different intensities of the spill, corresponding to a flux between $0.5 \mathrm{kHz} / \mathrm{cm}^{2}$ and 15 $\mathrm{kHz} / \mathrm{cm}^{2}$ in the central region of the beam spot.

For data analysis, tracks were first selected by asking for a single hit on both planes of the $\mathrm{X}-\mathrm{Y}$ hodoscope. A coincidence of $\mathrm{X}$ and $\mathrm{Y}$ strips was used to select a $1 \mathrm{~cm}^{2}$ spot at the beam center. RPC hits were defined by requiring a signal in a $40 \mathrm{~ns}$ time window, and the multi-hit TDC allowed us to record also the background hits in a $64 \mu \mathrm{s}$ window before the trigger. Clusters were formed by joining adiacent strips and from those we obtained the "raw" efficiency $\varepsilon_{\text {raw }}$. To correct for the effect of accidentals, we used the off-time hits over a $5 \mu$ interval, appropriately rescaled to the $40 \mathrm{~ns}$ window, and we determined the "accidental" efficiency, $\varepsilon_{a c c}$. The real efficiency was then computed from the formula: $1-\varepsilon_{\text {true }}=(1-$ $\left.\varepsilon_{\text {raw }}\right) /\left(1-\varepsilon_{a c c}\right)$. This correction was typically below $1 \%$.

Fig. 1 shows the efficiency curves for the SRPC and DRPC under different beam intensity conditions. The DRPC shows clearly better efficiency at large rate, with almost no visible drop in performance up to $10 \mathrm{kHz} / \mathrm{cm}^{2}$ and beyond. The SRPC shows some efficiency drop: at 9.8 $\mathrm{kV} \varepsilon$ decreases from $98 \%$ at $2 \mathrm{kHz} / \mathrm{cm}^{2}$ to $96 \%$ at $5 \mathrm{kHz} / \mathrm{cm}^{2}$, and to $90 \%$ at $15 \mathrm{kHz} / \mathrm{cm}^{2}$. Hit maps of the two detectors show a larger cluster size for the DRPC. At the beginning of the efficiency plateau, the average cluster size, $\langle H\rangle$, is about 1.7 strips for the SRPC, and twice as much for the DRPC. These values increase with the HV. Given the fact that the two detectors had different strip sizes and layout, it is possible that the larger cluster width of the DRPC could be reduced with a different strip design. At the moment, however, since a small cluster 
size is important for the trigger performance of $\mathrm{LHCb}$, the SRPC solution seems preferable, given the fact that good efficiency is obtainable also with this type of detector.

\section{GIF tests}

The GIF (Gamma Irradiation Facility) at CERN is based on a very intense (740 GBq) ${ }^{137} \mathrm{Cs}$ source. The GIF is used to test detectors under continuous photon load, with fluxes comparable to those expected by the large LHC detectors. LHCb operates under worse background conditions than ATLAS and CMS, so our setup was arranged to maximize the flux. Unfortunately we were not able to test the DRPC and only a triplet of identical SRPC was tested there.

The GIF setup is shown in Fig. 2. The source is placed in a protected area on the X5 SPS beam line. A system of filters can be used to adjust the intensity of the $662-\mathrm{keV}$ photons and to make it reasonably uniform over the area of the detectors. In this process the energy spectrum of the photons is considerably degraded. The triplet of SRPC was placed on a plane at $1 \mathrm{~m}$ from the GIF where the flux at minimum attenuation $(\mathrm{Att}=1)$ is given by $\Phi_{\gamma}=2.5 \cdot 10^{6}$ $\mathrm{Hz} / \mathrm{cm}^{2}$. Of course only a small fraction of this flux is detected by the RPC. A muon beam of low intensity (beam divergence about $1 \mathrm{mrad}$ ) is used to measure the efficiency of the RPC under varying load conditions. The procedure is the same as outlined previously in Sect. 3 .

To obtain an estimate of the detected photon flux, we measured the counting rate of the various strips on each plane. The rates were added together, and to correct for the overestimate due to the cluster size $>1$ we divided the result by the average cluster width $\langle H\rangle$ obtained for beam particles at the actual value of the HV: $R_{\gamma}=\sum R_{i} /\langle H\rangle$. When $R_{\gamma}$ is plotted vs. the $\mathrm{HV}$ value a plateau is visible. We took the rate measured at $10 \mathrm{kV}$ on the RPC which had the best plateau as the nominal photon rate. At Att=1 the measured flux was $3.1 \mathrm{kHz} / \mathrm{cm}^{2}$, corresponding to a sensitivity of the RPC to photons $\varepsilon_{\gamma} \cong 1.210^{-3}$. This appears to be somewhat smaller than previous estimates. The dependence of the flux is not linear in 1/Att, but rather proportional to $(1 / \mathrm{Att})^{0.76}$. The analysis of these data is still preliminary [5].

The efficiency curves for muons as a function of the HV, with GIF Att $=50$ and Att $=1$ are shown in Fig. 3 and 4 for the X and Y readout of the three RPCs. At Att $=1$ the onset of the plateau is displaced by about $400 \mathrm{~V}$ with respect to source off conditions. However the chambers still reach $>95 \%$ efficiency. At $10 \mathrm{kV}$ the currents drawn by the chambers were about $0.2,0.4$ and $0.3 \mathrm{~mA}$.

To improve efficiency a majority coincidence of 2 out of 3 planes has also been tested. Coincidences were made between the overlapping strips on the three planes. Efficiencies $>98 \%$ are reached in this case. Another advantage of the majority scheme is that the width of the clusters and the tails are reduced considerably.

\section{Conclusion}

Comparison of high-intensity data has shown that double-gap RPCs have better rate capability than single-gap, as expected. Single-gap have smaller cluster width. Beam and GIF results for the efficiency do not show a complete agreement, the detector being apparently more "loaded" by the source. This effect is being investigated.

The results have shown that the single-gap RPC can meet the LHCb requirements. With the majority scheme efficiencies $>98 \%$ were obtained at a photon flux of $3.1 \mathrm{kHz} / \mathrm{cm}^{2}$. 
Acknowledgements The authors are indebted to G. Iaselli and R. Santonico for providing the bakelite plates and for several discussions.

\section{References}

[1] LHCb Technical Proposal, CERN/LHCC 98-4, 1998.

[2] R. Cardarelli, A. Di Ciaccio and R. Santonico, Nucl. Instr. and Meth. in Phys. Res. A 333 (1993) 399; R. Cardarelli, V. Makeev, R. Santonico, Nucl. Instr. and Meth. in Phys. Res. A 382 (1996) 470.

[3] CMS Technical Proposal, CERN/LHCC 94-38, 1994.

[4] P. Camarri et al. Nucl. Instr. and Meth. in Phys. Res. A 414 (1998) 317.

[5] When scaled for the different distances, our results agree with those reported by M. Maggi et al, Proc. IV Int. Workshop on RPC and Related Detectors, Scientifica Acta Univ. Pavia, vol XIII n.2 (1998) 139. 
$\omega$

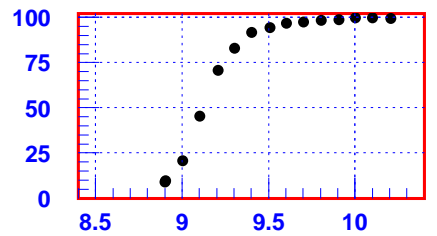

$\omega$

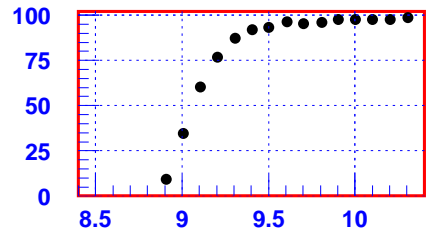

$\omega$

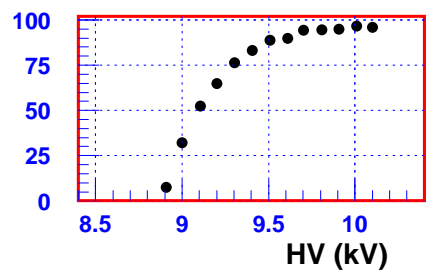

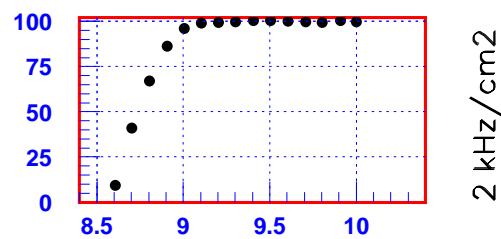
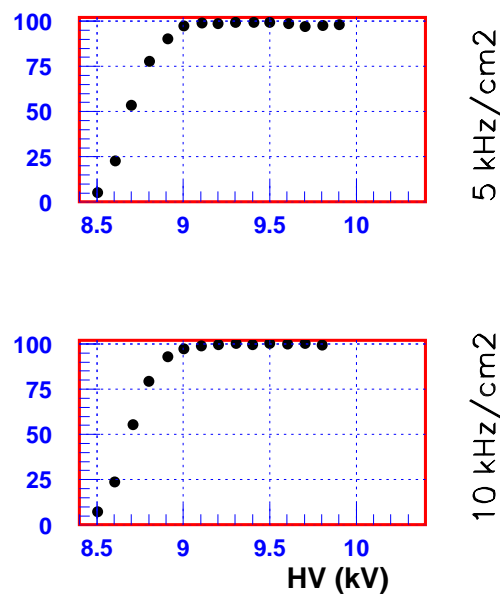

$$
\frac{\text { E }}{\frac{N}{x}}
$$

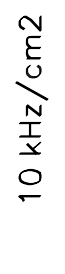

Figure 1 Efficiency curves for SRPC (left) and DRPC (right) at various beam intensities.

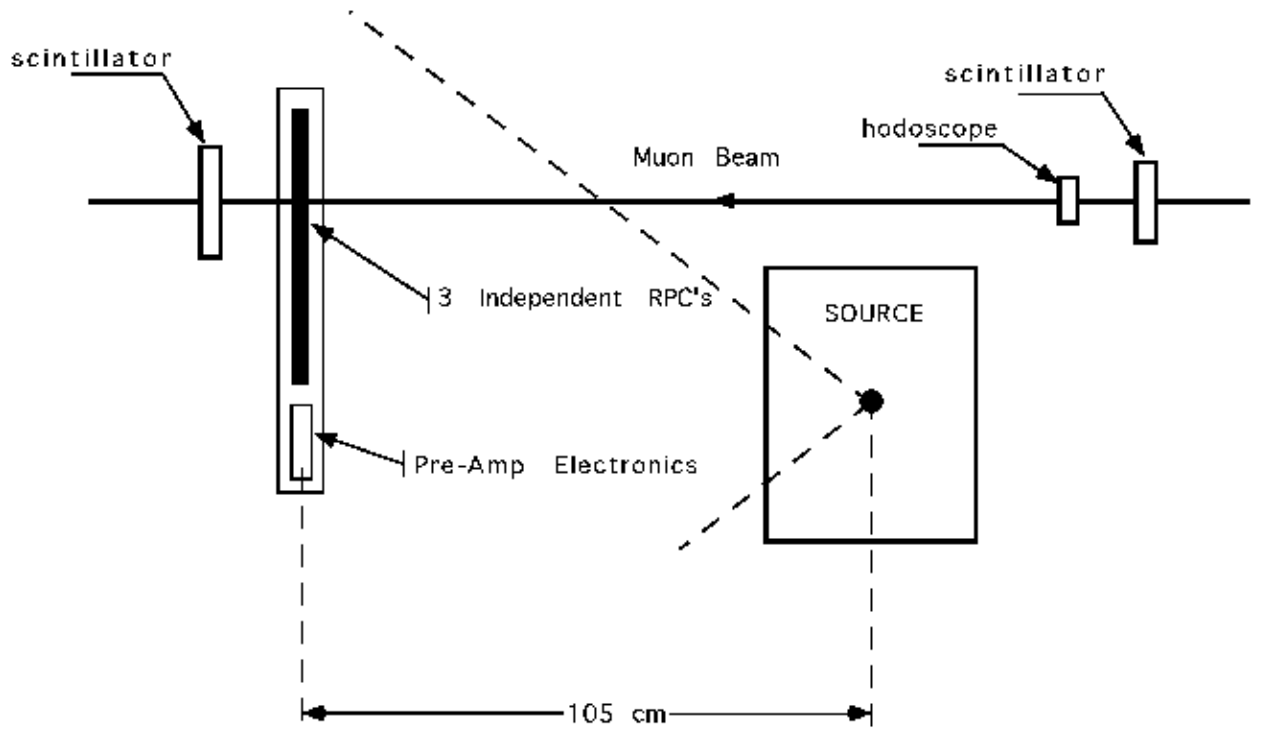

Figure 2 GIF test setup 
GIF Att $=50$
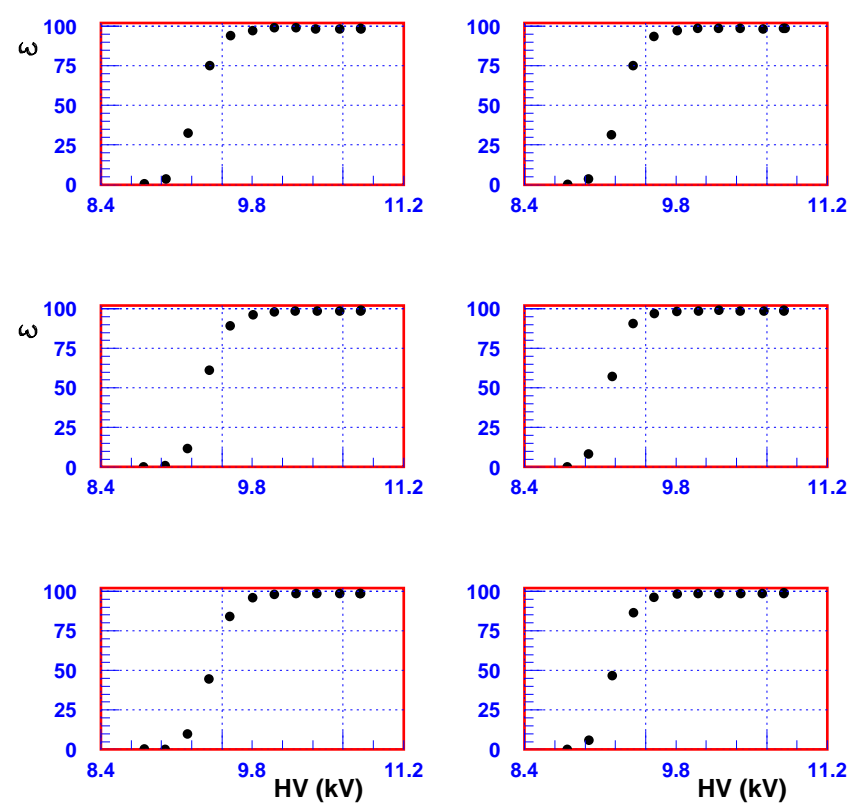

Figure 3 Efficiency curves for planes X (left) and Y (right) of the three SRPCs, for GIF attenuation 50 (flux $160 \mathrm{~Hz} / \mathrm{cm}^{2}$ ).
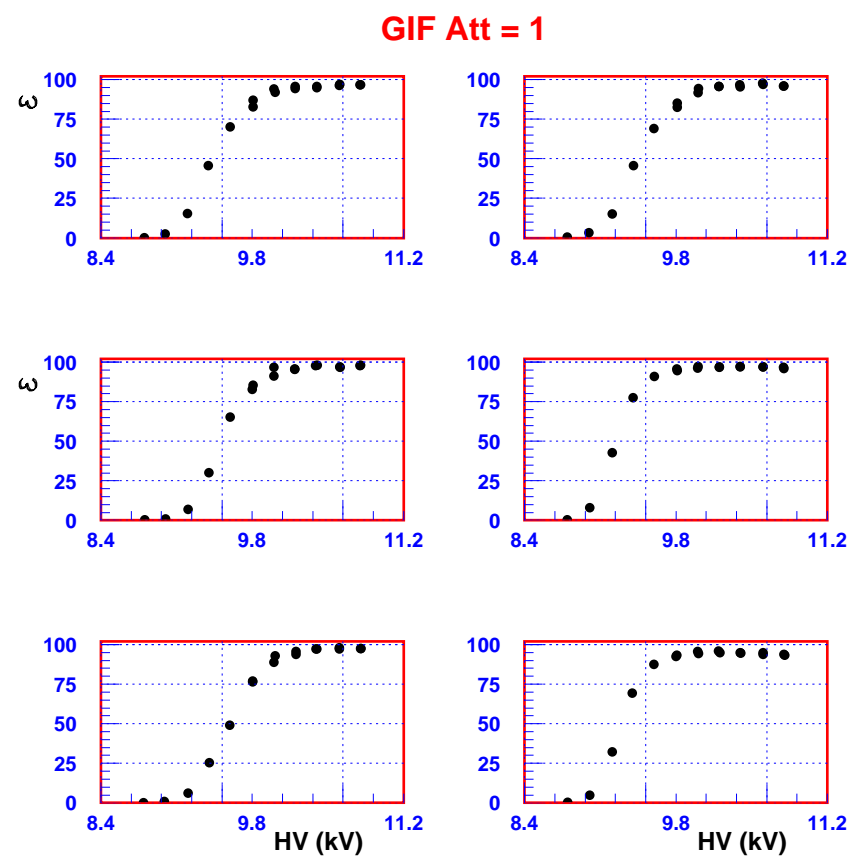

Figure 4 Efficiency curves for planes X (left) and Y (right) of the three SRPCs, for GIF attenuation 1 (flux $3.1 \mathrm{kHz} / \mathrm{cm}^{2}$ ). 